\title{
Spinal epidural hematomas: personal experience and literature review of more than 1000 cases
}

\author{
Maurizio Domenicucci, MD, ${ }^{1}$ Cristina Mancarella, MD, ${ }^{1}$ Giorgio Santoro, MD, ${ }^{1}$ \\ Demo Eugenio Dugoni, MD, ${ }^{1}$ Alessandro Ramieri, MD, ${ }^{2}$ Maria Felice Arezzo, MS, ${ }^{3}$ and \\ Paolo Missori, MD ${ }^{1}$
}

1Department of Neurology and Psychiatry, Neurosurgery, and ${ }^{3}$ Department of Methods and Models for Economics, Territory and Finance, "Sapienza" University of Rome; and 2Orthopedic Division, Don Gnocchi Foundation, Milan, Italy

\begin{abstract}
OBJECTIVE The goal of this study was to identify factors that contribute to the formation of acute spinal epidural hematoma (SEH) by correlating etiology, age, site, clinical status, and treatment with immediate results and long-term outcomes.
\end{abstract}

\begin{abstract}
METHODS The authors reviewed their series of 15 patients who had been treated for SEH between 1996 and 2012. In addition, the authors reviewed the relevant international literature from 1869 (when SEH was first described) to 2012, collecting a total of 1010 cases. Statistical analysis was performed in $959(95 \%)$ cases that were considered valid for assessing the incidence of age, sex, site, and clinical status at admission, correlating each of these parameters with the treatment results. Statistical analysis was also performed in $720(71.3 \%)$ cases to study the incidence of etiological factors that favor SEH formation: coagulopathy, trauma, spinal puncture, pregnancy, and multifactorial disorders. The clinical status at admission and long-term outcome were studied for each group. Clinical status was assessed using the Neuro-Grade (NG) scale.
\end{abstract}

RESULTS The mean patient age was 47.97 years (range 0-91 years), and a significant proportion of patients were male $(60 \%, p<0.001)$. A bimodal distribution has been reported for age at onset with peaks in the 2 nd and 6 th decades of life. The cause of the SEH was not reported in $42 \%$ of cases. The etiology concerned mainly iatrogenic factors (18\%), such as coagulopathy or spinal puncture, rather than noniatrogenic factors $(29 \%)$, such as genetic or metabolic coagulopathy, trauma, and pregnancy. The etiology was multifactorial in $11.1 \%$ of cases. The most common sites for SEH were C- 6 ( $n$ $=293,31 \%)$ and $\mathrm{T}-12(\mathrm{n}=208,22 \%)$, with maximum extension of 6 vertebral bodies in 720 cases $(75 \%)$. At admission, $806(84 \%)$ cases had moderate neurological impairment (NG 2 or 3), and only lumbar hematoma was associated with a good initial clinical neurological status (NG 0 or 1). Surgery was performed in 767 (80\%) cases. Mortality was greater in patients older than 40 years of age $(9 \% ; p<0.01)$. Sex did not influence any of these data $(p>0.05)$.

CONCLUSIONS Factors that contribute to the formation of acute SEH are iatrogenic, not iatrogenic, or multifactorial. The treatment of choice is surgery, and the results of treatment are influenced by the patient's clinical and neurological status at admission, age, and the craniocaudal site.

https://thejns.org/doi/abs/10.3171/2016.12.SPINE15475

KEY WORDS spinal epidural hematoma; outcome; review; coagulopathy; trauma; lumbar puncture; pregnancy

$\mathrm{S}$ PINAL epidural hematoma (SEH) is a relatively rare but disabling disease that was described for the first time by Jackson ${ }^{34}$ in 1869 . Available studies in the literature have reported series with small numbers of cases treated at a single institution and contain limited and irrelevant clinical and statistical data for an epidemiological study. ${ }^{6}$ Several reviews of the literature have also been conducted. ${ }^{29}$

Here, we report cases from our institution and from a broad review of the literature on SEH. We reviewed more than 1000 cases and statistically analyzed the general epi- demiological data, etiology, clinical status, treatment, and long-term outcome.

\section{Methods}

Between 1996 and 2012, 15 patients were treated for SEH in the Division of Neurosurgery of the Department of Neurology and Psychiatry at Sapienza University in Rome; we reviewed the data from these cases. In addition, a computer-aided search of MEDLINE was conducted using PubMed to obtain literature published between 1869 
and September 2012. The search was performed using the keywords "spinal epidural hematoma" and was limited to articles discussing SEH in humans and in the English, French, Italian, German, or Spanish languages. Reports in languages other than these (e.g., Chinese, Korean) were included only if the English-language abstract included comprehensive data for statistical analysis. Moreover, studies that reported a series of patients who were not described individually were excluded. We found 1010 cases, excluding postsurgical neoplastic SEH and SEH subsequent to arteriovenous malformations.

Table 1 summarizes the meaningful clinical data for the 1010 cases (i.e., our own cases and those from the literature). Statistical analysis of age, sex, anatomical level and site of the hematoma, clinical condition, and treatment results (Tables 2 and 3) was performed for 959 cases (95\%); 51 cases were excluded because of incomplete data. In addition to an assessment of each vertebra, we divided SEH localization into 5 specific locations: cervical, cervicothoracic, thoracic, thoracolumbar, and lumbar.

Clinical status at the time of the first observation and follow-up was assessed using a modified Neuro-Grade (NG) scale previously reported in other studies. ${ }^{19,20}$ This scale defines clinical status according to 4 grades: Grade 0 , no deficits; 1 , back pain, mild sensorimotor and/or sphincteric deficits; 2 , para- or tetraparesis; and 3, para- or tetraplegia. This simplified scale was used to standardize the clinical data in a pattern that allows statistical comparisons. In relation to treatment, the cases were divided into surgical (laminectomy or other surgical treatment for decompression of the hematoma) and nonsurgical cases.

With regard to etiology, cases were evaluated by classifying them as iatrogenic according to predisposing factors (drug-induced coagulopathy and spinal puncture), noniatrogenic (secondary to trauma, metabolic inherited coagulation disorder, and pregnancy), or multifactorial. Idiopathic cases were those for which it was explicitly stated that no diseases or events closely correlated with spinal epidural bleeding. The statistical analysis of the etiological factors involved 720 cases (71\%) after excluding 290 (29\%) cases for which these data were not reported (Table 4). A detailed study assessing neurological conditions at admission and the long-term results was performed in $195(90 \%)$ of the 216 cases affected by coagulopathy, 196 $(87.5 \%)$ of the 224 cases with trauma, and $62(68.9 \%)$ of the 90 cases in which spinal puncture was performed.

Patients who exhibited the following abnormal hematological indices were considered to be affected by coagulopathy: international normalized ratio $(>1.2)$, partial thromboplastin time ( $>50$ seconds), partial thromboplastin $(>13$ seconds), and platelets $(<150,000 / \mu \mathrm{l})$. Patients receiving high doses of anticoagulant therapy but with no reported abnormality in hematological indices were included in this group. Patients receiving antiplatelet therapy with no abnormality in hematological indices were considered separately. Cases were excluded if a description of the hematological indices or the anticoagulant therapy was missing.

The term "spinal puncture" was used to include all invasive procedures of the spine performed, such as spinal or epidural anesthesia, diagnostic lumbar puncture, or epidural catheter insertion. The cases with spinal trauma were studied in 2 distinct groups: major trauma resulting from an event with grave consequences to the spine, and minor trauma due to aggravation because of a daily activity that is in itself not detrimental.

We performed a statistical analysis of proportions to assess the significance of prevalence as a $p$ value. We used nonparametric tests, (the Kolmogorov-Smirnov test and Mann-Whitney U-test) to compare outcomes between 2 independent groups.

\section{Results}

The distribution of publications concerning SEH over the years was hyperbolic. Half of the cases were reported in the literature during the first 123 years (18691992) since SEH was first described, whereas the other half were reported during the next 19 years, after CT and MRI examinations became routine in clinical practice.

\section{Age and Sex}

This study comprised 573 (60\%) males and 386 (40\%) females ( $<<0.001$, Table 2 ). The overall mean age was 47.97 years (range 0-91 years). The mean age of males was 47.59 years (range $0-91$ years), and that for females was 51.04 years (range $0-90$ years, $p<0.05$ compared with males). The age distribution curve in decades was bimodal with peak prevalence in the 2nd and 6th decades. Therefore, we separated patients into 2 groups: $0-40$ years and 41-91 years. No significant sex differences were observed $(p>0.05)$.

\section{Localization}

Effects of the presence of SEH were reported for all vertebrae, but with different frequencies, and the data assumed a bimodal trend. An increased peak incidence occurred at C-6 $(\mathrm{n}=293,31 \%)$ and T-12 $(\mathrm{n}=208,22 \%)$, and no significant difference $(\mathrm{p}>0.05)$ was found between the sexes. SEH extended between 1 and 6 vertebrae in 720 cases $(75 \%)$ and between 2 or 3 in 347 cases (36\%). The extension of SEH most often involved the thoracic vertebrae $(\mathrm{n}=254,26 \%)$. In 44 cases $(4.60 \%)$ the hematoma was associated with the entire vertebral column, the sacral segment, or separate vertebrae.

\section{Clinical Presentation}

In the 959 cases considered valid for this study (Table 2) 22 patients (2\%) had an NG score of 0 and 806 patients (84\%) had an NG score of 2 or $3(n=385,40 \%$ NG $2 ; n=$ $421,44 \%$ NG 3 ). The small percentage of patients with an NG score of 0 was significant ( $p<0.05$ ), as was the high percentage of cases with an NG score of 2 or 3 . Based on the Kolmogorov-Smirnov test and Mann-Whitney U-test, this distribution of NG score was unaffected by sex $(p>$ $0.05)$ or age when using 40 years as the cutoff ( $p>0.05)$.

Evaluation of the clinical condition in relation to the site of the hematoma, based on the Kolmogorov-Smirnov test, showed that lumbar localization (133 cases) significantly differed from the others in the prevalence of NG 1 $(n=56,42 \%)$, with a low prevalence of NG $3(n=22,17 \%)$ compared with the other sites. 


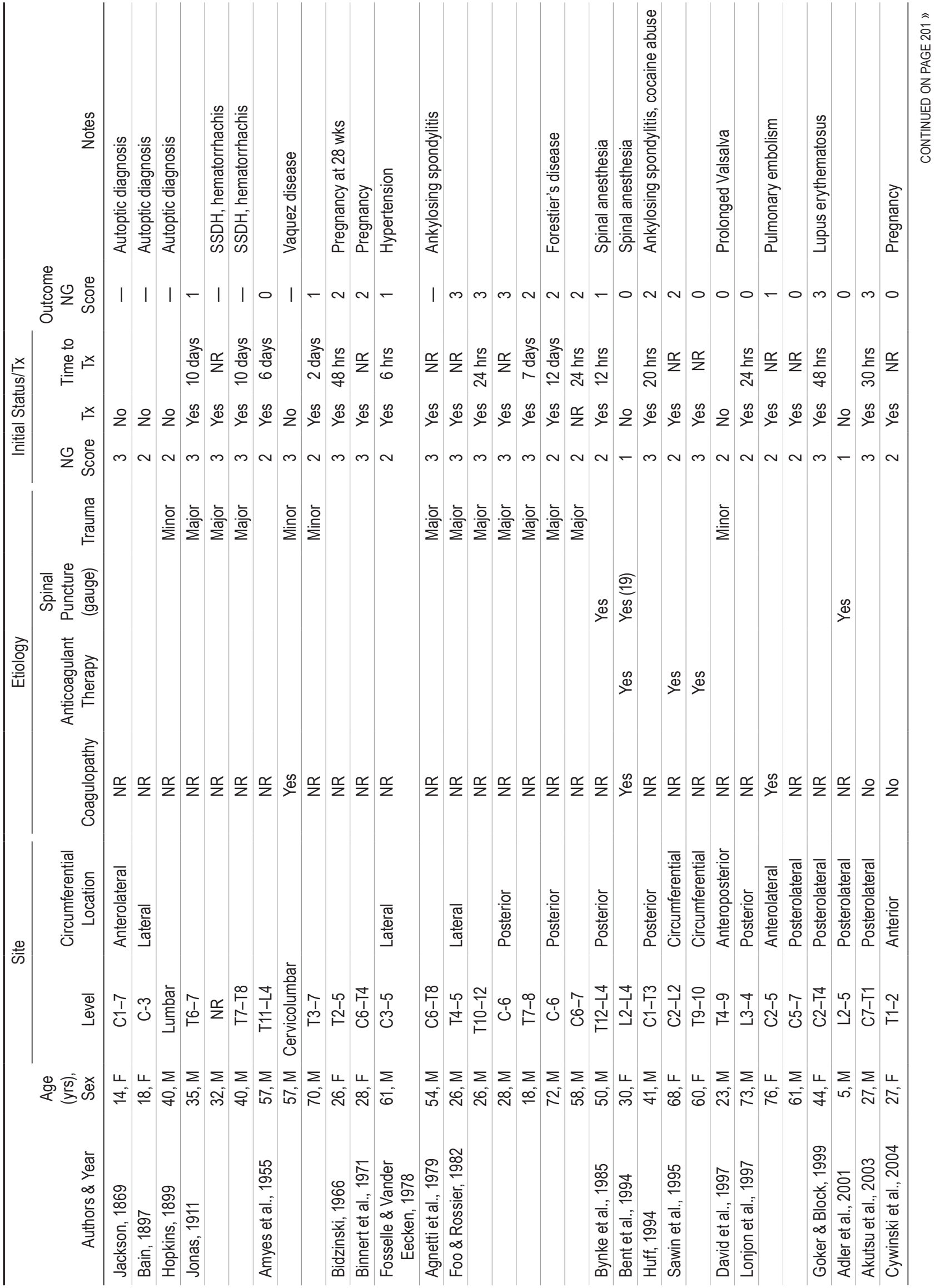




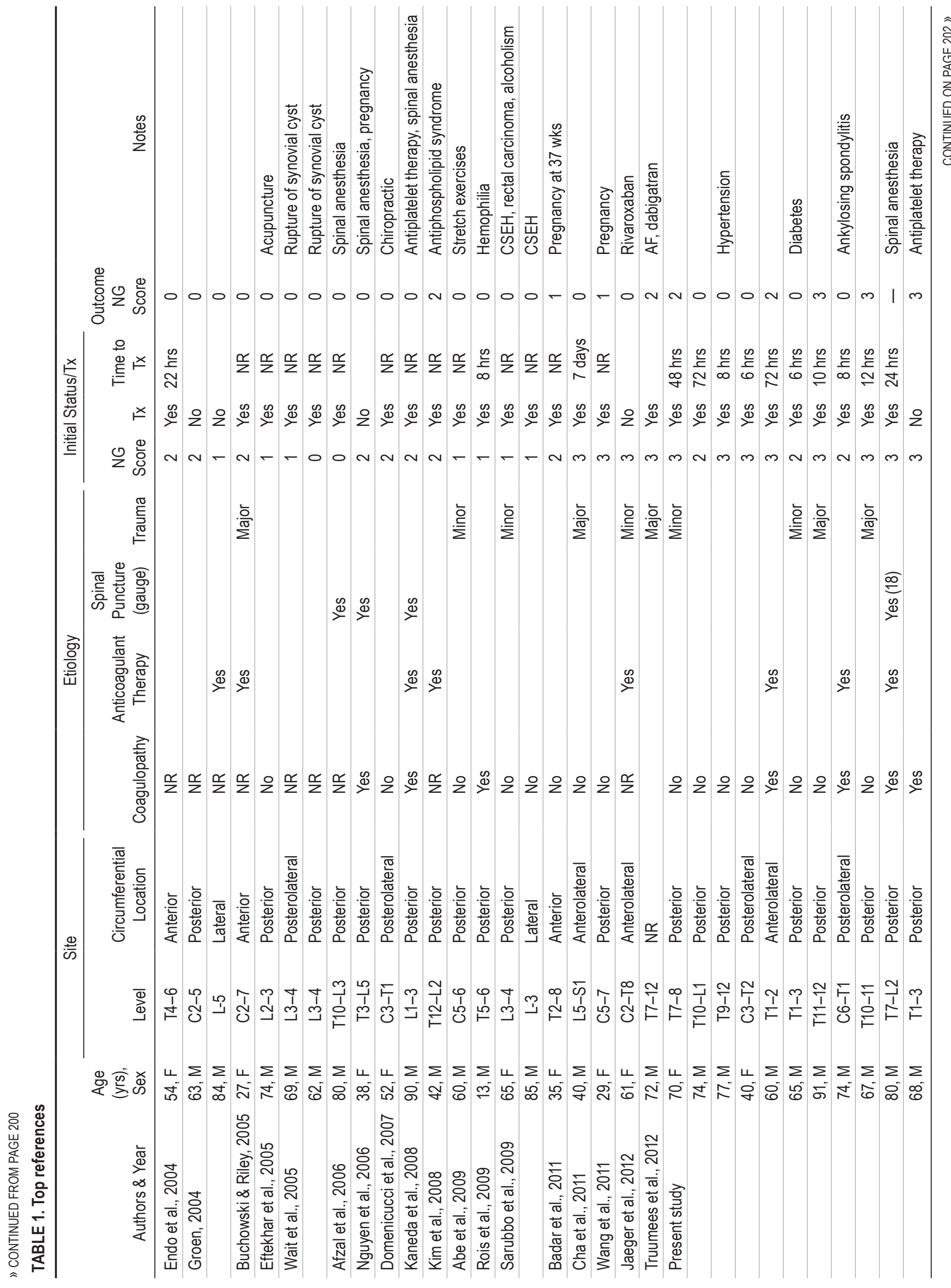




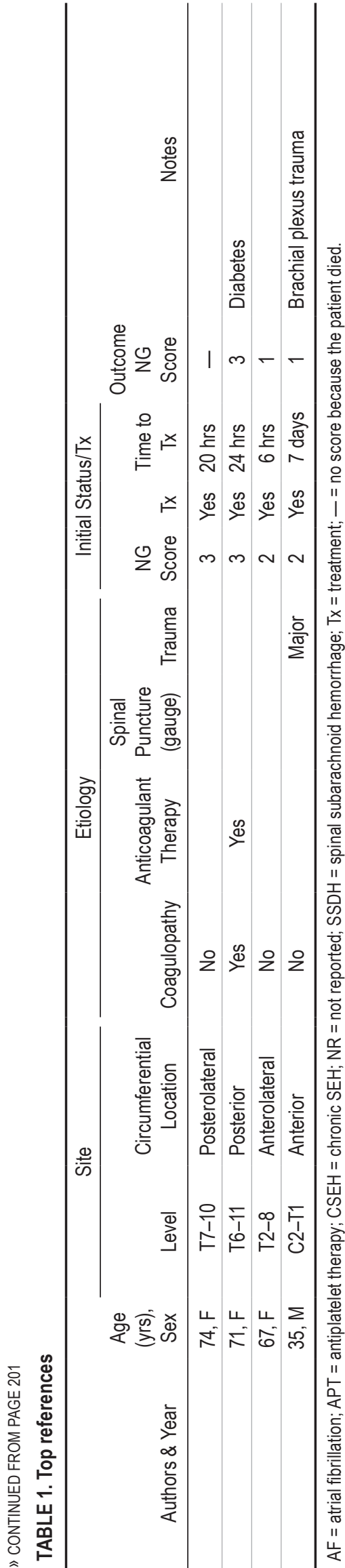

TABLE 2. General data of 959 cases of SEH in relation to sex, age, site, and treatment

\begin{tabular}{|c|c|c|c|c|c|}
\hline \multirow[b]{2}{*}{ Variable } & \multirow{2}{*}{$\begin{array}{c}\text { No. of } \\
\text { Patients } \\
(\%)\end{array}$} & \multicolumn{4}{|c|}{ NG Score at Admission } \\
\hline & & 0 & 1 & 2 & 3 \\
\hline $\begin{array}{l}\text { No. of } \\
\text { patients }\end{array}$ & 959 & $22(2)$ & $131(14)$ & $385(40)$ & $421(44)$ \\
\hline Total & 959 & $22(2)$ & $131(14)$ & $385(40)$ & $421(44)$ \\
\hline Op & $767(80)$ & $6(1)$ & $83(11)$ & $304(40)$ & $373(49)$ \\
\hline Nonop & $192(20)$ & $16(8)$ & $47(24)$ & $81(42)$ & $48(25)$ \\
\hline \multicolumn{6}{|l|}{ Sex } \\
\hline Male & $573(60)$ & $12(2)$ & $87(15)$ & $227(40)$ & $247(43)$ \\
\hline Op & $453(79)$ & $3(1)$ & $55(12)$ & $175(39)$ & $220(49)$ \\
\hline Nonop & $120(21)$ & $9(8)$ & $32(27)$ & $52(43)$ & $27(22)$ \\
\hline Female & $386(40)$ & $10(3)$ & $44(11)$ & $158(41)$ & $174(45)$ \\
\hline Op & $314(81)$ & $3(1)$ & $29(9)$ & $129(41)$ & $153(49)$ \\
\hline Nonop & $72(19)$ & $7(10)$ & $15(21)$ & $29(40)$ & $21(29)$ \\
\hline \multicolumn{6}{|l|}{ Age in yrs } \\
\hline $0-40$ & $331(35)$ & $8(2)$ & $50(15)$ & $124(37)$ & $148(45)$ \\
\hline Op & $261(79)$ & $0(0)$ & $29(11)$ & $96(37)$ & $136(52)$ \\
\hline Nonop & $70(21)$ & $8(11)$ & $22(31)$ & $28(40)$ & $12(17)$ \\
\hline $41-91$ & $628(65)$ & $14(2)$ & $80(13)$ & $261(42)$ & $273(43)$ \\
\hline Op & $506(81)$ & $6(1)$ & $55(11)$ & $209(41)$ & $238(47)$ \\
\hline Nonop & $122(19)$ & $8(7)$ & $25(20)$ & $52(43)$ & $35(29)$ \\
\hline \multicolumn{6}{|l|}{ Site } \\
\hline Cervical & $203(21)$ & $5(2)$ & $23(11)$ & $100(49)$ & 75 (37) \\
\hline $\begin{array}{l}\text { Cervico- } \\
\text { thoracic }\end{array}$ & $188(20)$ & $1(1)$ & $12(6)$ & $80(43)$ & $95(51)$ \\
\hline Thoracic & $254(26)$ & $4(2)$ & $18(7)$ & $82(32)$ & $150(59)$ \\
\hline $\begin{array}{l}\text { Thoraco- } \\
\text { lumbar }\end{array}$ & $137(14)$ & $6(4)$ & $13(10)$ & $52(38)$ & $66(48)$ \\
\hline Lumbar & $133(14)$ & $5(4)$ & $56(42)$ & $50(38)$ & $22(17)$ \\
\hline Other & $44(5)$ & $1(2)$ & $9(20)$ & $21(48)$ & $13(30)$ \\
\hline
\end{tabular}

The initial clinical status is indicated by the NG scale.

Considering surgically treated and nonsurgically treated groups separately, 6 of the 767 surgically treated patients (1\%) had an NG score of 0 and $83(11 \%)$ an NG score of 1 . Sixteen of 192 patients without surgery (8\%) had an NG score of 0 , and 47 (24\%) had an NG score of 1. The percentage of surgically treated patients with an NG score of 3 was $49 \%(n=373)$, higher than the percentage of nonsurgically treated patients $(25 \%, \mathrm{n}=48)$ with an $\mathrm{NG}$ score of 3 . The average NG score was significantly different between the 2 groups $(\mathrm{p}<0.01)$. The number of surgically treated cases with an NG score of 2 was $304(40 \%)$, which is comparable to the $81(42 \%)$ nonsurgically treated patients with an NG score of 2.

\section{Treatment}

A total of $767(80 \%)$ patients underwent surgery, and $192(20 \%)$ did not. Moreover, $79 \%(\mathrm{n}=453)$ of males and $81 \%(\mathrm{n}=314)$ of females underwent surgical treatment. In addition, $79 \%(\mathrm{n}=261)$ of patients younger than 40 years of age and $81 \%(n=506)$ of patients older than 40 years of 
TABLE 3. Outcome in 959 cases of SEH in relation to sex, age, site, and treatment

\begin{tabular}{|c|c|c|c|c|c|c|}
\hline \multirow[b]{2}{*}{ Variable } & \multirow{2}{*}{$\begin{array}{c}\text { No. of } \\
\text { Patients (\%) }\end{array}$} & \multicolumn{5}{|c|}{ NG Score at Outcome } \\
\hline & & 0 & 1 & 2 & 3 & Dead \\
\hline No. of patients & 959 & $441(46)$ & $138(14)$ & $190(20)$ & $121(13)$ & $69(7)$ \\
\hline Op & 767 & $312(41)$ & $115(15)$ & $183(24)$ & $115(15)$ & $42(5)$ \\
\hline Nonop & 192 & $129(67)$ & $23(12)$ & $7(4)$ & $6(3)$ & $27(14)$ \\
\hline \multicolumn{7}{|l|}{ Sex } \\
\hline Male & 573 & $274(48)$ & $73(13)$ & $116(20)$ & $70(12)$ & $40(7)$ \\
\hline Op & 453 & $188(42)$ & $61(13)$ & $111(24)$ & $67(15)$ & $26(6)$ \\
\hline Nonop & 120 & $86(72)$ & $12(10)$ & $5(4)$ & $3(2)$ & $14(12)$ \\
\hline Female & 386 & $167(43)$ & $65(17)$ & $74(19)$ & $51(13)$ & $29(8)$ \\
\hline Op & 314 & $124(40)$ & $54(17)$ & $72(23)$ & $48(15)$ & $16(5)$ \\
\hline Nonop & 72 & $43(60)$ & $11(15)$ & $2(3)$ & $3(4)$ & $13(18)$ \\
\hline \multicolumn{7}{|l|}{ Age in yrs } \\
\hline $0-40$ & 331 & $173(52)$ & $35(11)$ & $68(20)$ & $42(13)$ & $13(4)$ \\
\hline Op & 261 & $118(45)$ & $33(13)$ & $65(25)$ & $39(15)$ & $6(2)$ \\
\hline Nonop & 70 & $55(79)$ & $2(3)$ & $3(4)$ & $3(4)$ & $7(10)$ \\
\hline $41-91$ & 628 & $268(43)$ & $103(16)$ & $122(19)$ & $79(13)$ & $56(9)$ \\
\hline Op & 506 & $194(39)$ & $82(16)$ & $118(23)$ & $76(15)$ & $36(7)$ \\
\hline Nonop & 122 & 74 (61) & $21(17)$ & $4(3)$ & $3(2)$ & $20(17)$ \\
\hline \multicolumn{7}{|l|}{ Site } \\
\hline Cervical & 203 & $103(51)$ & $28(14)$ & $44(22)$ & $11(5)$ & $17(8)$ \\
\hline Cervicothoracic & 188 & $84(45)$ & $35(19)$ & $32(17)$ & $22(11)$ & $15(8)$ \\
\hline Thoracic & 254 & $97(38)$ & $37(15)$ & $54(21)$ & $52(20)$ & $14(6)$ \\
\hline Thoracolumbar & 137 & $45(33)$ & $19(14)$ & $37(27)$ & $25(18)$ & $11(8)$ \\
\hline Lumbar & 133 & $88(66)$ & $15(11)$ & $18(14)$ & $7(5)$ & $5(4)$ \\
\hline Other & 44 & $24(55)$ & $4(9)$ & $5(11)$ & $4(9)$ & $7(16)$ \\
\hline
\end{tabular}

age underwent surgical treatment, showing that patient sex and age did not significantly $(\mathrm{p}>0.05)$ affect the choice of conservative or surgical treatment.

\section{Outcome}

The clinical results of long-term follow-up in a total of 959 cases considered to be valid were evaluated (Table 3 ). A total of 579 cases $(60 \%)$ had an outcome NG score of 0 or 1 , and 311 cases (32\%) had an outcome with an NG score of 2 or 3 . Mortality was revealed in 69 cases $(7 \%)$. Patients older than 40 years of age had a mortality of $9 \%$ $(\mathrm{n}=56)$, significantly $(\mathrm{p}<0.01)$ higher than the mortality $(4 \%, \mathrm{n}=13)$ in patients younger than 40 years of age or younger. The distribution of NG scores was not significantly different between males and females.

In relation to neurological status at admission, of the 152 patients who had an NG score of $0(n=22)$ or $1(n=130)$, $13(8.6 \%)$ had a persistent NG score of 1 independent of the treatment received. Of the 384 patients with an NG score of 2 at presentation, 301 patients (78.4\%) improved in their neurological status to an NG score of 0 or 1 , and the mortality rate was $3.12 \%(n=12)$. Among the 385 cases with an NG score of 2 at presentation, mortality was $1.64 \%(\mathrm{n}=$ $5)$ for the surgery group and significantly higher $(\mathrm{p}<0.01)$ for the nonsurgery group $(8.64 \%, \mathrm{n}=7 / 81)$. The recovery of clinical conditions in the NG 0 and 1 groups was similar between the 2 groups ( $60.1 \%$ and $75 \%$, respectively). Of the
420 cases with an NG score of 3 at follow-up, 124 (29.5\%) recovered to an NG score of 0 or $1,119(28.3 \%)$ had a persistent NG score of 3, and $56(13.3 \%)$ died. Among the patients with an NG score of 3, the mortality rates were $9.9 \%$ $(\mathrm{n}=37)$ for the surgical group and significantly higher $(\mathrm{p}<$ $0.01)$ for the nonsurgical group $(40.4 \%, \mathrm{n}=19)$.

With regard to prognosis of all patients in relation to the site of the hematoma, $88(66 \%)$ of 133 patients with lumbar SEH had an NG score of 0 at follow-up, and only 7 patients (5\%) had an NG score of 3, achieving the best result at follow-up. A test of the equality of proportions established that the lumbar site had the best performance $(p<0.05)$. The thoracic site had a significantly worse result $(\mathrm{p}<0.05)$ compared with the others, with an NG score of 3 in 52 of 254 patients (20\%).

\section{Etiology}

Table 4 summarizes the etiology of SEH. In 290 cases (29\%) the data were not reported or were incomplete; the remaining 720 cases (75\%) were divided into 185 (18\%) with iatrogenic etiological factors, such as drugs or spinal puncture, 292 (29\%) with noniatrogenic factors, such as genetic or metabolic coagulopathy, trauma, and pregnancy, and 108 (11\%) with multiple combined etiological factors. Etiological causes were not reported in 135 cases (13\%); these cases were defined as idiopathic. No significant prevalence of a specific etiological cause was found. 
TABLE 4. Etiology of SEH

\begin{tabular}{cc}
\hline \multicolumn{1}{c}{ Etiology } & No. of Patients $(\%)$ \\
\hline latrogenic factors & $185(18)$ \\
\hline Coagulopathy & $90(49)$ \\
\hline Anticoagulant therapy & $69(77)$ \\
\hline Antiplatelet therapy & $21(23)$ \\
\hline Antiplatelet therapy & $48(26)$ \\
\hline Spinal puncture & $47(25)$ \\
\hline Noniatrogenic factors & $292(29)$ \\
\hline Coagulopathy & $66(23)$ \\
\hline Genetic & $27(41)$ \\
\hline Metabolic & $20(30)$ \\
\hline Not defined & $19(29)$ \\
\hline Trauma & $199(68)$ \\
\hline Major & $114(57)$ \\
\hline Minor & $76(38)$ \\
\hline Not defined & $9(5)$ \\
\hline Pregnancy & $27(9)$ \\
\hline Multifactorial & $108(11)$ \\
\hline Coagulopathy + spinal puncture & $39(36)$ \\
\hline Coagulopathy + trauma & $21(19)$ \\
\hline Spinal puncture + trauma & $4(4)$ \\
\hline Other & $44(41)$ \\
\hline Idiopathic & $135(13)$ \\
\hline NR & $290(29)$ \\
\hline Total & 1010 \\
\hline &
\end{tabular}

\section{Coagulopathy}

Coagulopathy was present in 216 cases (21.4\%): 90 $(41.7 \%)$ caused by drug therapy, $47(21.8 \%)$ related to noniatrogenic causes, 19 (8.8\%) with unidentified coagulopathy, and $60(27.8 \%)$ in which the coagulopathy was associated with other factors, such as spinal puncture (n $=39,18 \%)$ or trauma $(\mathrm{n}=21,9.7 \%)$. In 48 cases $(4.8 \%)$ antiplatelet therapy was reported as the single etiological factor without any alteration in the hematological indices.

The age distribution of patients with coagulopathy differed significantly $(\mathrm{p}<0.01)$ from that reported in the general case studies of SEH: $18(8 \%)$ patients in the 0 - to 40-year age group and 198 (92\%) patients in the 41- to 91-year age group. Considering only the 47 patients with genetic and/or metabolic coagulopathy, the proportion of males was significant $(\mathrm{p}<0.01)$ with a male/female ratio of $36: 11$. The average age of males (in these categories) was 30 years. Excluding this minority of cases, the percentages of surgical and nonsurgical cases, the incidence of SEH according to sex, and the site of SEH with coagulopathy coincided with the general case series, as did the distribution of cases in relation to NG input, mortality, and follow-up.

\section{Trauma}

We identified $224(22.2 \%)$ patients with posttraumatic SEH, 114 (50.9\%) with major trauma, and 76 (33.9\%) with minor trauma. Twenty-one cases $(9.4 \%)$ were associated with a coagulopathy and $4(1.8 \%)$ with spinal puncture. In 9 cases (4\%) the trauma was not defined. Male sex was more prevalent $(\mathrm{p}<0.01)(168$ [75\%] vs 56 [25\%] female patients). The mean age of these patients was 41 years, which is significantly lower $(\mathrm{p}<0.01)$ than that of the general population with SEH. In patients with posttraumatic $\mathrm{SEH}$ at admission, clinical status, as well as long-term outcome, was similar to that of patients in the general case series, with no difference between patients with major or minor trauma.

\section{Spinal Puncture}

In 113 cases $(11.2 \%)$ the SEH was associated with a spinal puncture, which was the only etiological factor in 47 cases (41.5\%). Spinal puncture was associated with a coagulopathy in 39 cases (34.5\%) and with trauma in 4 cases $(3.5 \%)$. The significantly involved sites $(\mathrm{p}<0.05)$ were lumbar and thoracolumbar in 69 patients $(61 \%)$, followed by thoracic in 30 patients (26.5\%), and these sites differed significantly $(\mathrm{p}<0.05)$ from those in the general case series series. The remaining 14 lesions (12.4\%) were located in the cervical region. At admission, most patients with an SEH in the thoracic region $(67 \% ; \mathrm{n}=20)$ had an NG score of 3. At the long-term follow-up, 9 (30\%) patients remained in this condition; the scores in the remaining 11 patients improved but returned to an NG score of $0(7 \%)$ in only 2 patients. However, 26 (38\%) of the 69 patients with an SEH at lumbar and thoracolumbar sites had an NG score of 3 at admission, and all recovered to a score of 0 at follow-up.

\section{Pregnancy}

Thirty-three patients (3.3\%) with SEH were pregnant. In 27 cases $(82 \%)$ pregnancy was the only predisposing factor, and all of the lesions were localized in the thoracic region with the exception of one that was cervical. In 6 cases (18\%) a spinal puncture was performed. In cases with a favorable initial NG score $(\mathrm{n}=0$ [NG score of 0 ]; $\mathrm{n}=4$ [NG score of 1]) the outcome was an NG score of 0 in all cases, and 3 of the patients with an initial NG score of 1 underwent surgery. The 8 patients with an initial NG score of 2 at the follow-up improved to an NG score of 0 , and 7 underwent surgery. Among 21 patients with an initial NG score of 3, a favorable outcome (NG score of 0 or 1) occurred in 10 patients $(48 \%)$. No deaths were noted, in contrast to the $7 \%$ mortality rate in the general case series.

\section{Multifactorial}

In 108 cases (11\%), more etiological factors suitable to promote the formation of SEHs were reported. In 55\% of these cases a coagulopathy was present, associated with spinal puncture in 39 cases (36\%) and trauma in 21 cases $(19 \%)$. The reporting of etiological factors in the remaining cases was occasional and varied.

\section{Idiopathic}

In 135 patients (13\%) the SEH was not associated with any type of predisposing factor; therefore, these lesions were identified as idiopathic. Among patients with idiopathic lesions with an initial NG score of $0(n=5,3.7 \%)$ 
and $1(\mathrm{n}=38,28 \%)$, the outcome score was 0 in all patients. In 46 patients with an initial NG score of 2 , the outcome score was 0 or 1 in 34 patients (74\%). In 45 patients with an initial NG score of 3, the outcome score was 2 or 3 in 24 patients $(53 \%)$, and 8 patients died (17\%). These longterm results of idiopathic cases in relation to the initial NG score reflect the outcomes in the overall cases. In relation to treatment, $49 \%$ of patients with an initial NG score of $0,87 \%$ with a score of $1,95 \%$ with a score of 2 , and $89 \%$ with a score of 3 underwent surgery, which is significantly more than in the overall series.

\section{Discussion}

Jackson $^{34}$ described the first case of SEH. In 1869, he reported a cervical epidural collection of blood that had been diagnosed during autopsy in a young woman who had presented with progressive weakening of the upper and lower limbs and subsequently died due to a loss of respiratory function. The first case to be successfully surgically treated was reported by Jonas ${ }^{36}$ in 1911, although Lonjon et al. ${ }^{41}$ mistakenly attributed it to Bain, ${ }^{8}$ who in 1897 had only studied the disease at autopsy, as had Jackson before and Hopkins $^{32}$ in 1899. Jonas ${ }^{36}$ described the surgical procedure as a laminectomy of T-6 and T-7 to remove an epidural blood clot; he called this pathology "hematorrhachis." The patient, a farmer, was paraplegic before the surgical procedure, and at follow-up walked with support.

Until 1950 only 19 cases had been reported in the literature. From 1951 to 1970 , approximately 100 cases had been published, whereas the majority of the works that refer to SEHs were published after 1971, with more than 900 cases reported until 2011. The distribution of publications concerning SEH highlights the growing interest in this topic, but it may also correlate with improved diagnostic tools, such as CT in 1979 and MRI in 1987.

The first surgical series was from 1911, when Jonas ${ }^{36}$ reported 3 cases of SEH caused by spine fractures. The largest series reported to date, and the most consistent, was a series of 23 cases described by Liu et al. ${ }^{40}$ in 2008 . The most extensive reviews thus far have been those of Kreppel et al. ${ }^{39}$ in 2004 and Groen, ${ }^{29}$ reporting 461 cases of SEH in the context of 613 cases of spinal hematoma and 548 cases of spontaneous SEH, including 64 nonsurgical cases, respectively.

The present study is a retrospective review based on case reports from the literature and our own series of 15 patients with SEH. In the absence of an original series related to a unique institution, due to the rarity of the disease in question, we used the international literature to collect a sufficient number of cases from which we could obtain reliable statistical data. The greatest difficulty in collecting data was the lack of standardization of data regarding the initial status and outcomes of the patients, which is why we adopted the NG scale used in other works. ${ }^{19,20}$

$\mathrm{SEH}$ was more prevalent in males (three-fifths of cases), with an older mean age than female patients. The distribution of cases according to age showed that SEH was more prevalent in the 2nd and 6th decades of life, which was similar between sexes. The craniocaudal localization of spinal SEH referred to all segments, but the most com- mon sites affected were the C-6 and T-12 vertebrae, with rare involvement of the sacral canal. The most affected site was the thoracic spine, accounting for one-fourth of cases. SEH mainly extended over 3 consecutive vertebrae, and the majority of cases had a maximum length of 6 vertebral bodies. Cases involving a single vertebra or the entire length of the spine were extremely rare.

Neurological examination at admission found that most cases were severe with para- or quadriparesis (NG Score 2) or serious spinal paralysis (NG Score 3); only $14 \%$ had minor deficits (NG Score 1). In a few cases, there was clinical evidence that the only pain was in the spine (NG Score 0 ). These findings did not change in relation to patient sex or age. Patients with hematomas in the lumbar spine presented in a significantly better neurological condition than those with hematomas at other sites.

In our review, we found that surgery was preferred to conservative treatment, and this choice was not influenced by the age or sex of the patient. The determining factor in the choice of treatment was the patient's neurological status. One-third of the patients who did not undergo surgery had a moderate deficit, whereas approximately half of the patients with para- or quadriparesis underwent surgery, as conservative treatment was reserved for patients with good initial status.

Long-term outcomes were good, with NG scores of 0 or 1 in two-thirds of the patients, but significant differences were found at follow-up in relation to the initial clinical status, patient age, site of the hematoma, and treatment received; patient sex did not affect the results. A worse initial neurological status correlated with a worse result; the majority of cases with NG scores of 0 or 1 had favorable follow-up, and only $8.5 \%$ persisted with a minimum deficit. In patients with severe symptoms (NG score of 2 or 3), healing or a small disabling deficit was detected in $78.4 \%$ and $28.3 \%$ of patients, respectively. Patient age was decisive in regard to mortality. Death occurred less frequently in younger patients regardless of the treatment. Prognosis was most favorable for lumbar SEH, and the thoracic SEH had the worst long-term results. The sites were equivalent in terms of mortality.

About one-tenth of the examined cases were idiopathic. In most cases, a major cause could be determined, such as a major trauma, spinal puncture, or coagulopathy, or a predisposing factor, such as a minor trauma, pregnancy, or antiplatelet therapy. However, none of these determinants or predisposing factors had greater prevalence over the other. We examined these etiological factors individually to detect special features. We did not define any groups under the term "spontaneous" because, in the literature, this term is used to describe patients with idiopathic $\mathrm{SEH}^{27}$ as well as those with coagulopathy, ${ }^{37}$ female patients who were pregnant, ${ }^{16}$ patients undergoing treatment with antiplatelet drugs, ${ }^{38}$ those with vascular malformations, ${ }^{25,27}$ or patients with a combination of these conditions, ${ }^{3}$ leading to some confusion in terminology. We agree with Lonjon et al. ${ }^{41}$ that the definition "spontaneous spinal epidural hematoma," which is often used in the literature, is ambiguous, but the expression "non-traumatic spinal epidural hematoma" used by those authors is equally confusing, as it does not exclude patients with bleeding disorders or vascular malformation. 
Therefore, we used the term "idiopathic," as it optimally identifies the group of cases in which there are no potential etiologies causing or predisposing the patient to SEH. ${ }^{5}$

\section{SEH and Coagulopathy}

The group of patients with SEH associated with an alteration in hematological indices was heterogeneous and included mainly iatrogenic cases of patients with anticoagulant or antiplatelet treatment, but also cases with metabolic disease, such as severe liver disease, and genetic causes, such as congenital deficiency of factor VIII. In 1995, Sawin et al. ${ }^{47}$ described the first case of SEH during thrombolysis with tissue plasminogen activator. A minority of patients included in this group ( $<5 \%$ of cases) were started on a regimen of antiplatelet therapy without any hematological alteration in the indices. The prevalence in SEH with coagulopathy in patients older than 40 years of age (almost $90 \%$ of cases) may be related to anticoagulant and antiplatelet therapies generally reserved for those in adulthood and senile age. These cases have a clinical and therapeutic course and immediate and long-term outcome that overlap with those of the general SEH patient population. The data in the genetic and metabolic coagulopathy groups, in which there is a male prevalence, are different, and the mean age is younger, probably due to the early onset of genetic diseases. ${ }^{45}$

\section{SEH and Trauma}

The literature does not clearly distinguish between SEH caused by major or minor trauma or evaluate their possible clinical diversity. ${ }^{14}$ In accordance with Foo and Rossier, ${ }^{26}$ we classified traumatic events to the spine as major according to their ability to produce an osteoligamentous lesion, or as minor when the traumatic event was caused by physical activities with important dynamic stress, such as after stretching exercises, ${ }^{1}$ daily work, ${ }^{11}$ or chiropractic spinal manipulation therapy. ${ }^{21}$ Trauma is the leading cause of SEH in the group with noniatrogenic etiology, accounting for more than two-thirds of the cases in this group. The male prevalence in this group and the lower average age of males compared with females is related to the different work activities and sports between sexes. ${ }^{26}$

\section{SEH and Spinal Puncture}

Spinal puncture for diagnostic ${ }^{2}$ or therapeutic practices and spinal anesthesia, ${ }^{9}$ with or without placement of an intradural or epidural catheter, ${ }^{13}$ is reported in approximately one-tenth of cases of SEH. The prevalence of lumbar or thoracolumbar localization correlates with the preferred site for spinal puncture and insertion of a needle and/or catheter. Neurological status at admission and the long-term outcome are much more favorable if the SEH is located in the lumbar region for both conservative and surgical treatment. The thoracic site is characterized by worse clinical conditions at admission and worse longterm results.

\section{SEH and Pregnancy}

SEH is a rare event during pregnancy and has been reported in only 33 cases after the first report by Bidzin- $\mathrm{ski}^{10}$ in 1966 . We agree with Wang et al. ${ }^{50}$ that the etiology remains unknown. It is probably multifactorial, and one of the main predisposing factors remains venous bleeding related to the epidural venous plexus, which is affected by pressure variability in the chest cavity and abdomen during efforts or breaths. ${ }^{15,30}$ This theory is confirmed by the fact that the localization is almost exclusively thoracic; SEH manifests in the last weeks of pregnancy when the abdominal space is larger and there are no other etiological factors. The therapeutic strategy is linked not only to the neurological status of the patient, but also the stage of fetal maturation, and the approach is multidisciplinary. All but one of the patients underwent surgery.

\section{Idiopathic SEH}

In approximately one-tenth of cases, no etiological factors were described at admission. The statistical analysis showed that this group had a greater percentage of surgical cases independent of the NG score at admission. This therapeutic strategy was probably the most accepted because there was no pathological contraindication to the treatment. However, there was no difference with regard to the general data and outcome.

\section{Multiple Etiological Factors and SEH}

One-tenth of cases involved a combination of several etiological factors that cause SEH. Three-fifths of these patients were affected by coagulopathy, which is associated mainly with a major or minor trauma and spinal puncture. SEH after a lumbar puncture is one of the most dangerous complications. Manchikanti et al. ${ }^{42}$ reported the main guidelines and recommendations to be considered in order to limit the risks of bleeding associated with interventional practices in patients receiving anticoagulant therapy.

A rare association also affects SEH resulting from spinal puncture in pregnant women; the 6 reported cases are all related to anesthesia practices during childbirth and may be related to vascular congestion, giving a reason for the onset of these hematomas, ${ }^{15,30}$ as well as alterations in coagulation ${ }^{43}$ especially in term pregnancies.

\section{Rare Cases of SEH}

Rare and exceptional causes of SEH described in the literature include the ingestion of large amounts of garlic, ${ }^{24}$ trumpet playing, ${ }^{17}$ chiropractic spinal manipulation therapy, ${ }^{21}$ acupuncture, ${ }^{22}$ cocaine abuse, ${ }^{33}$ lupus erythematosus, ${ }^{28}$ ankylosing spondylitis, ${ }^{4}$ immobilization of a "hangman's fracture,"12 rupture of a synovial cyst ${ }^{49}$ or arachnoid cyst, ${ }^{23}$ and pseudogout. ${ }^{18}$ These can all be associated with one of the etiological types that we considered: coagulopathy, trauma, spinal puncture, and metabolic disease.

\section{Controversial SEH}

In a few cases, there were doubts regarding the etiology and nature of the hematoma. Twenty-three cases of chronic SEH were reported, and they were described in relation to the surgical macroscopic characteristics of hematomas and often lasted for several weeks or months. Moreover, these hematomas tended to localize in the lumbar region with good long-term prognosis. The literature shows that 
diagnosis is mainly surgical,7,31 and in more recent studies histology of the capsule of the alleged hematoma has not been performed, ${ }^{44,46}$ even though it could change the diagnosis. ${ }^{45}$ Gundry and Heithoff ${ }^{31}$ reported an additional 18 similar cases, all associated with disc protrusion in the lumbar region, and 14 of them presented with a capsule. The authors seemed to describe chronic hematomas in patients with prolonged clinical histories, even up to a year. Yet, in no case was the capsule histologically examined. The capsule was described as adhering to the discs and/ or ligamentous structures. Because of uncertainties in the etiology of these SEHs, they could be chronic hemorrhagic synovial cysts, and they were not included in our series. With regard to the first reports of SEH during treatment with oral anticoagulants, such as factor Xa inhibitors, ${ }^{35,48}$ there are uncertainties regarding the alteration in hematological indices and, therefore, these cases cannot be included among those associated with coagulopathy.

Data analysis allowed us to confirm that the treatment of SEH remains controversial. The etiology, particularly laboratory data on coagulation, plays an important role in the decision-making process when choosing a conservative or surgical treatment. In the presence of an increased risk of bleeding, which occurs in approximately one-third of the patients tested, decisions should be made carefully. The risk of bleeding must be corrected pharmacologically. Surgery is the recommended treatment in case of severe neurological symptoms, particularly for NG scores of 2 , or in the case of clinical deterioration. These patients, as well as those undergoing conservative treatment, should undergo follow-up with careful clinical and laboratory observation. SEH in patients undergoing antiplatelet therapy with normal coagulation parameters presents a dilemma. These cases cannot be classified as coagulopathic, but patients should still be treated as potentially suffering from a deficiency in coagulation unless future studies exclude this hypothesis.

Because of incomplete information and the inability to obtain significant data, our work has omitted an evaluation of findings regarding $\mathrm{SEH}$ in relation to the timing of surgery, the circumferential extension and location of the hematoma, and the size of the needles that caused a hematoma during spinal puncture. Moreover, the long period taken into account did not allow for the collection of homogeneous data because, during the first years we reviewed, there was no opportunity to be so detailed. The limitations of our work are that it is retrospective and the studies that we reviewed appeared in other specialist journals, such as surgery, anesthesiology, and hematology. This factor could have distorted the data, particularly regarding the prevalence of surgical treatments for SEH and the etiology of the disease. Only a future large-scale prospective study can confirm or refute the reported data.

\section{Conclusions}

SEH is a rare event, but it occurs frequently enough to be observed at least once in the clinical practice of every neurosurgeon and spine specialist. A sufficiently large, single-institution series to obtain relevant statistical data has not yet been described, requiring the use of a review of published cases to collect enough data to statistically analyze the etiology, treatment, and outcome of the disease.

Etiological factors that favor the onset of SEH can be iatrogenic, such as drug-induced coagulopathy, lumbar puncture, trauma, or genetic coagulation disorders, and play an important role in the choice of treatment and longterm results. In a small number of cases identified as idiopathic, no causes were shown. The treatment of choice is surgery, and the results of treatment are influenced by the patient's clinical condition and neurological status at admission, age, and the hematoma location. Further largescale studies may confirm or vary from the statistical data in this literature review.

\section{Acknowledgments}

We thank Lattanzi Natalino, a computer scientist who graduated in physics, who helped us to collect data and build tables.

\section{References}

1. Abe T, Nagamine Y, Ishimatsu S, Tokuda Y: Spinal epidural hematoma after stretch exercise: a case report. Am J Emerg Med 27:902.e1-902.e2, 2009

2. Adler MD, Comi AE, Walker AR: Acute hemorrhagic complication of diagnostic lumbar puncture. Pediatr Emerg Care 17:184-188, 2001

3. Afzal A, Hawkins F, Rosenquist RW: Epidural hematoma in a patient receiving epidural analgesia and LMWH after totalknee arthroplasty. Reg Anesth Pain Med 31:480, 2006

4. Agnetti V, Monaco F, Mutani R: Post-convulsive spinal epidural haematoma in ankylosing spondylitis. Eur Neurol 18:230-233, 1979

5. Akutsu H, Sugita K, Sonobe M, Matsumura A: A case of nontraumatic spinal epidural hematoma caused by extradural varix: consideration of etiology. Spine J 3:534-538, 2003

6. Amyes EW, Vogel PJ, Raney RB: Spinal cord compression due to spontaneous epidural hemorrhage; report of three cases. Bull Los Angel Neuro Soc 20:1-8, 1955

7. Badar F, Kirmani S, Rashid M, Azfar SF, Yasmeen S, Ullah E: Spontaneous spinal epidural hematoma during pregnancy: a rare obstetric emergency. Emerg Radiol 18:433-436, 2011

8. Bain W: A case of hematorrachis. BMJ 2:455, 1897

9. Bent U, Gniffke S, Reinbold WD: [An epidural hematoma following single shot epidural anesthesia.] Anaesthesist 43:245-248, $1994(\mathrm{Gr})$

10. Bidzinski J: Spontaneous spinal epidural hematoma during pregnancy. Case report. J Neurosurg 24:1017, 1966

11. Binnert D, Thierry A, Michiels R, Soichot P, Perrin M: [Presentation of a new case of spontaneous spinal extradural hematoma observed during labor.] J Med Lyon 52:1307-1309, $1971(\mathrm{Fr})$

12. Buchowski JM, Riley LH III: Epidural hematoma after immobilization of a "hangman's" fracture: case report and review of the literature. Spine J 5:332-335, 2005

13. Bynke O, Johansson KE, Sökjer H: [Intraspinal epidural hematoma - an unusual complication in epidural anesthesia.] Lakartidningen 82:1772-1774, 1774, 1985 (Swedish)

14. Cha JR, Park KB, Ko SH: Post-traumatic lumbar epidural hematoma with neurology: report of 1 case. Asian Spine J 5:130-132, 2011

15. Clemens HJ: [A contribution to the histology of the internal spinal venous plexus.] Z Mikrosk Anat Forsch 67:183-189, 1961 (Ger)

16. Cywinski JB, Parker BM, Lozada LJ: Spontaneous spinal epidural hematoma in a pregnant patient. J Clin Anesth 16:371-375, 2004

17. David S, Salluzzo RF, Bartfield JM, Dickinson ET: Spontane- 
ous cervicothoracic epidural hematoma following prolonged valsalva secondary to trumpet playing. Am J Emerg Med 15:73-75, 1997

18. deSouza RM, Uff C, Galloway M, Dorward NL: Spinal epidural hematoma caused by pseudogout: a case report and literature review. Global Spine J 4:105-108, 2014

19. Domenicucci M, Ramieri A, Ciappetta P, Delfini R: Nontraumatic acute spinal subdural hematoma: report of five cases and review of the literature. J Neurosurg 91 (1 Suppl):65-73, 1999

20. Domenicucci M, Ramieri A, Paolini S, Russo N, Occhiogrosso G, Di Biasi C, et al: Spinal subarachnoid hematomas: our experience and literature review. Acta Neurochir (Wien) 147:741-750, 2005

21. Domenicucci M, Ramieri A, Salvati M, Brogna C, Raco A: Cervicothoracic epidural hematoma after chiropractic spinal manipulation therapy. Case report and review of the literature. J Neurosurg Spine 7:571-574, 2007

22. Eftekhar B, Ketabchi E, Ghodsi M, Esmaeeli B: Lumbar epidural hematoma due to lumbar acupunctures. Neurol India 53:245-246, 2005

23. Endo H, Takahashi T, Shimizu H, Tominaga T: Thoracic intradural arachnoid cyst associated with surgical removal of epidural hematoma-case report. Neurol Med Chir (Tokyo) 44:607-610, 2004

24. Fedder SL: Spinal epidural hematoma and garlic ingestion. Neurosurgery 27:659, 1990

25. Foo D, Chang YC, Rossier AB: Spontaneous cervical epidural hemorrhage, anterior cord syndrome, and familial vascular malformation: case report. Neurology 30:308-311, 1980

26. Foo D, Rossier AB: Post-traumatic spinal epidural hematoma. Neurosurgery 11:25-32, 1982

27. Fosselle E, Vander Eecken H: Idiopathic spinal epidural haematoma. Lancet 2:42, 1978

28. Goker B, Block JA: Spinal epidural hematoma complicating active systemic lupus erythematosus. Arthritis Rheum 42:577-578, 1999

29. Groen RJM: Non-operative treatment of spontaneous spinal epidural hematomas: a review of the literature and a comparison with operative cases. Acta Neurochir (Wien) 146:103-110, 2004

30. Groen RJM, Groenewegen HJ, van Alphen HA, Hoogland PV: Morphology of the human internal vertebral venous plexus: a cadaver study after intravenous Araldite CY 221 injection. Anat Rec 249:285-294, 1997

31. Gundry CR, Heithoff KB: Epidural hematoma of the lumbar spine: 18 surgically confirmed cases. Radiology 187:427431, 1993

32. Hopkins SD: Extradural spinal meningeal hemorrhage. NY Med J 70:296-297, 1899

33. Huff JS: Spinal epidural hematoma associated with cocaine abuse. Am J Emerg Med 12:350-352, 1994

34. Jackson R: Case of spinal apoplexy. Lancet 2:5-6, 1869

35. Jaeger M, Jeanneret B, Schaeren S: Spontaneous spinal epidural haematoma during Factor Xa inhibitor treatment (Rivaroxaban). Eur Spine J 21 (Suppl 4):S433-S435, 2012

36. Jonas AF: Spinal fractures, opinions based on observations of sixteen operations. JAMA 9:859-863, 1911

37. Kaneda T, Urimoto G, Suzuki T: Spinal epidural hematoma following epidural catheter removal during antiplatelet therapy with cilostazol. J Anesth 22:290-293, 2008

38. Kim WJ, Hong YK, Yoo WH: Epidural hematoma mimicking transverse myelitis in a patient with primary antiphospholipid syndrome. Rheumatol Int 28:709-712, 2008

39. Kreppel D, Antoniadis G, Seeling W: Spinal hematoma: a literature survey with meta-analysis of 613 patients. Neurosurg Rev 26:1-49, 2003

40. Liu Z, Jiao Q, Xu J, Wang X, Li S, You C: Spontaneous spi- nal epidural hematoma: analysis of 23 cases. Surg Neurol 69:253-260, 2008

41. Lonjon MM, Paquis P, Chanalet S, Grellier P: Nontraumatic spinal epidural hematoma: report of four cases and review of the literature. Neurosurgery 41:483-487, 1997

42. Manchikanti L, Falco FJ, Benyamin RM, Caraway DL, Kaye $\mathrm{AD}$, Helm S II, et al: Assessment of bleeding risk of interventional techniques: a best evidence synthesis of practice patterns and perioperative management of anticoagulant and antithrombotic therapy. Pain Physician 16 (2 Suppl):SE261SE318, 2013

43. Nguyen L, Riu B, Minville V, Chassery C, Catalaa I, Samii $\mathrm{K}$ : [Epidural hematoma after hemorrhagic shock in a parturient.] Can J Anaesth 53:252-257, 2006 (Fr)

44. Ramieri A, Domenicucci M, Seferi A, Paolini S, Petrozza V, Delfini R: Lumbar hemorrhagic synovial cysts: diagnosis, pathogenesis, and treatment. Report of 3 cases. Surg Neurol 65:385-390, 2006

45. Rois PV, López MR, de Vergara BC, de la Lama Zaragoza A, García JG, Uxo JM: Spinal epidural hematoma in hemophilic children: controversies in management. Childs Nerv Syst 25:987-993, 995, 2009

46. Sarubbo S, Garofano F, Maida G, Fainardi E, Granieri E, Cavallo MA: Spontaneous and idiopathic chronic spinal epidural hematoma: two case reports and review of the literature. Eur Spine J 18:1055-1061, 2009

47. Sawin PD, Traynelis VC, Follett KA: Spinal epidural hematoma following coronary thrombolysis with tissue plasminogen activator. Report of two cases. J Neurosurg 83:350-353, 1995

48. Truumees E, Gaudu T, Dieterichs C, Geck M, Stokes J: Epidural hematoma and intraoperative hemorrhage in a spine trauma patient on Pradaxa (dabigatran). Spine (Phila Pa 1976) 37:E863-E865, 2012

49. Wait SD, Jones FD, Lonser RR, Lee KS: Symptomatic epidural hematoma caused by lumbar synovial cyst rupture: report of two cases and review of the literature. Neurosurgery 56:E1157, 2005

50. Wang P, Xin XT, Lan H, Chen C, Liu B: Spontaneous cervical epidural hematoma during pregnancy: case report and literature review. Eur Spine J 20 (Suppl 2):S176-S179, 2011

\section{Disclosures}

The authors report no conflict of interest concerning the materials or methods used in this study or the findings specified in this paper.

\section{Author Contributions}

Conception and design: Domenicucci. Acquisition of data: Mancarella, Santoro, Dugoni, Ramieri. Analysis and interpretation of data: Mancarella, Santoro, Dugoni. Drafting the article: Mancarella. Critically revising the article: Domenicucci. Reviewed submitted version of manuscript: Domenicucci, Missori. Statistical analysis: Arezzo. Study supervision: Missori.

\section{Supplemental Information \\ Online-Only Content}

Supplemental material is available with the online version of the article.

Supplemental Table 1. https://thejns.org/doi/suppl/10.3171/ 2016.12.SPINE15475.

\section{Correspondence}

Cristina Mancarella, Department of Neurological and Psychiatric Sciences, University "Sapienza," viale del policlinico 155, Rome 00161, Italy. email: cristina.mancarella@gmail.com. 